\title{
Leakage-Resilient Chosen-Ciphertext Secure Public-Key Encryption from Hash Proof System and One-Time Lossy Filter
}

\author{
Baodong Qin ${ }^{1,2}$ and Shengli Liu ${ }^{1, \star}$ \\ 1 Department of Computer Science and Engineering, Shanghai Jiao Tong University, \\ Shanghai 200240, China \\ 2 College of Computer Science and Technology, Southwest University of Science and \\ Technology, Mianyang 621010, China \\ \{qinbaodong, slliu\}@sjtu.edu.cn
}

\begin{abstract}
We present a new generic construction of a public-key encryption (PKE) scheme secure against leakage-resilient chosen-ciphertext attacks (LR-CCA), from any Hash Proof System (HPS) and any onetime lossy filter (OT-LF). Efficient constructions of HPSs and OT-LFs from the DDH and DCR assumptions suggest that our construction is a practical approach to LR-CCA security. Most of practical PKEs with LR-CCA security, like variants of Cramer-Shoup scheme, rooted from Hash Proof Systems, but with leakage rates at most 1/4-o(1) (defined as the ratio of leakage amount to secret-key size). The instantiations of our construction from the DDH and DCR assumptions result in LR-CCA secure PKEs with leakage rate of $1 / 2-o(1)$. On the other hand, our construction also creates a new approach for constructing IND-CCA secure (leakage-free) PKE schemes, which may be of independent interest.
\end{abstract}

Keywords: Public-key encryption, leakage-resilience, chosen-ciphertext security, hash proof system.

\section{Introduction}

Research on leakage-resilient cryptography is motivated by those side-channel attacks [17, in which a significant fraction of the secret key $S K$ is leaked to the adversary. Cryptosystems proved secure in the traditional model may suffer from these key-leakage attacks, as shown in [17. This fact leads to design and security proof of a variety of leakage-resilient cryptosystems, including stream ciphers [1430, block ciphers [12, digital signatures 2015, public key encryption [27 1 2 23 3 ], identity-based encryption [247/16], etc.

Leakage Oracle, Bounded-Leakage Model and Leakage Rate. Sidechannel attacks characterized by key leakage can be formalized in a general

\footnotetext{
* Supported by the National Natural Science Foundation of China (Grant No. 61170229, 61373153), the Specialized Research Fund for the Doctoral Program of Higher Education (Grant No. 20110073110016), and the Scientific innovation projects of Shanghai Education Committee (Grant No. 12ZZ021).
} 
framework [1] with a leakage oracle $\mathcal{O}_{S K}^{\lambda, \kappa}(\cdot)$ : the adversary queries arbitrary efficiently computable functions $f_{i}:\{0,1\}^{*} \rightarrow\{0,1\}^{\lambda_{i}}$ of the secret key $S K$ repeatedly and adaptively, and the leakage oracle responds with $f_{i}(S K)$. The bounded-leakage model limits the total amount of information about $S K$ leaked by the oracle to a bound $\lambda$ during the life time of the cryptosystem. This model is simple and powerful, but a thorough understanding of this model is essential to those more complicated models [4. If a cryptosystem is secure against the above key-leakage attacks, we call it $\lambda$-leakage-resilient ( $\lambda$-LR, for short). The leakage rate is defined as the ratio of $\lambda$ to the secret key size, i.e., $\lambda /|S K|$.

Leakage-Resilient CCA Security and Hash Proof System. In the keyleakage scenario of public key encryption (PKE), leakage-resilient security against chosen-plaintext attacks (LR-CPA) is characterized by the indistinguishability between the encryptions of two plaintexts (of equal length) chosen by any Probabilistic Polynomial-Time (PPT) adversary, who is given access to a key-leakage oracle. If the adversary is equipped with a decryption oracle as well, with restriction that the challenge ciphertext is refused by the decryption oracle and the leakage oracle stops working after the generation of the challenge ciphertext, the notion becomes leakage-resilient security against chosen-ciphertext attacks (LRCCA). Naor-Yung paradigm applies to LR-CCA security [27]. It achieves leakage rate of $1-o(1)$, but the simulation-sound Non-Interactive Zero-Knowledge (ssNIZK) proof is far from practical. It was later improved by Dodis et al. [11] with true-simulation extractable NIZK (tSE-NIZK), but the construction is still not practical. Recently, Galindo et al. [16] constructed an identity-based encryption (IBE) scheme with master key-dependent chosen-plaintext (mKDM-sID-CPA) security based on the decisional linear assumption over bilinear groups. They suggested that their mKDM-sID-CPA secure IBE scheme is also master key leakage resilient with rate $1-o(1)$, hence can be transformed into a LR-CCA secure PKE scheme with leakage rate $1-o(1)$ by applying the CHK transform [6]. However, their claim that the mKDM-sID-CPA secure IBE scheme is also master key leakage resilient was not supported by any rigorous proof.

Hash Proof Systems (HPSs), due to Cramer and Shoup [9], have long been served as the most practical approach to PKEs with IND-CCA security. They are also intrinsically LR-CPA secure, and a HPS based on the DDH assumption (and its $d$-Linear variant) was proved to be LR-CPA secure with leakage rate of $1-o(1)$ [27]. As to LR-CCA security, however, the HPS approach to INDCCA security is inherently limited to leakage rate below $1 / 2$, as pointed out by Dodis et al. 11. Recall that to achieve IND-CCA security, Cramer and Shoup [9] proposed to use two independent HPSs, one is a smooth HPS to mask and hide the plaintext, and the other is a universal ${ }_{2}$ HPS used to verify whether the ciphertext is well-formed. Hence two independent secret keys are involved in the construction, and either one, if totally leaked, will kill the LR-CCA security. That is why the leakage rate must be less than $1 / 2$.

Prior constructions of PKE with LR-CCA security from HPSs enjoy great efficiency, but suffer from low leakage rate. The variants [27/26] of CramerShoup DDH-based scheme [8] achieve leakage rate of $1 / 6-o(1)$, which was later 
improved to $1 / 4-o(1)$ 25. To the best of our knowledge, no constructions from HPSs are known to be LR-CCA secure with leakage rate of $1 / 2-o(1)$. The question is: can we find a new way to construct LR-CCA secure PKEs which are not only as practical as HPS but also with reasonable high leakage rates (like $1 / 2-o(1)) ?$

Our Contributions. We propose a new generic construction of PKE with LRCCA security from a Hash Proof System (HPS) and a one-time lossy filter (OT$\mathrm{LF}$ ). The new primitive, one-time lossy filter (OT-LF), is a weak version of lossy algebraic filter [19], and we show how to construct OT-LFs from the DDH and DCR assumptions. In the generic construction of LR-CCA secure PKE, the HPS is used to generate an encapsulated key $K$, which is not only used to mask the plaintext, but also used in the OT-LF to verify the well-formedness of ciphertexts. OT-LF helps to obtain a higher leakage rate, compared to the constructions solely from HPSs.

- We give instantiations of PKEs with LR-CCA security under the DDH (DCR) assumption, by combining an efficient construction of DDH (DCR)based OT-LF and DDH (DCR)-based HPS. The leakage rate is as high as $1 / 2-o(1)$.

- In case of no leakage on secret key at all, the leakage-free version of our construction opens another practical approach to IND-CCA security, as compared to the HPS-based construction by Cramer and Shoup.

Overview of Our Techniques. Different from the HPS-based approach to CCA-security, in which a universal 2 hash proof system is employed to reject ill-formed ciphertexts, we use a one-time lossy filter (OT-LF) to do the job. OT-LF is a simplified version of lossy algebraic filter, which was introduced by Hofheinz [19] recently to realize key-dependent chosen-ciphertext security [5]. The concept of OT-LF is similar to (chameleon) all-but-one lossy trapdoor function 3123 . But it does not require efficient inversion. Roughly, a OT-LF is a family of functions indexed by a public key $F p k$ and a tag $t=\left(t_{a}, t_{c}\right)$. A function $\operatorname{LF}_{F p k, t}(\cdot)$ from that family maps an input $X$ to a unique output. For a fixed public key, the set of tags contains two computationally indistinguishable disjoint subsets, namely the subset of injective tags and the subset of lossy ones. If tag $t=\left(t_{a}, t_{c}\right)$ is injective, then so is the corresponding function $\operatorname{LF}_{F p k, t}(\cdot)$. If the tag is lossy, the output of the function reveals only a constant amount of information about its input $X$. For any $t_{a}$, there exists a lossy tag $\left(t_{a}, t_{c}\right)$ such that $t_{c}$ can be efficiently computed through a trapdoor Ftd. Without this trapdoor, however, it is hard to generate a new lossy tag even with the knowledge of one lossy tag. Trapdoor Ftd and lossy tag are only used for the security proof.

Roughly speaking, a hash proof system HPS is a key-encapsulation mechanism. Given public key $p k$, an element $C \in \mathcal{V}$ and its witness $w$, the encapsulated key is given by $K=\operatorname{HPS} . \operatorname{Pub}(p k, C, w)$. With secret key $s k$, decapsulation algorithm HPS.Priv $(s k, C)$ recovers $K$ from $C \in \mathcal{V}$. If $C \in \mathcal{C} \backslash \mathcal{V}$, the output of HPS.Priv $(s k, C)$ has a high min-entropy even conditioned on $p k$ and $C$. The 
hardness of subset membership problem requires that elements in $\mathcal{V}$ are indistinguishable from those in $\mathcal{C} \backslash \mathcal{V}$.

In our construction, the secret key is just $s k$ from the HPS, and the HPS and OT-LF are integrated into a ciphertext $C T$,

$$
C T=\left(C, \quad s, \Psi=\operatorname{Ext}(K, s) \oplus M, \quad \Pi=\operatorname{LF}_{F p k, t}(K), t_{c}\right),
$$

via $K=\operatorname{HPS} . \operatorname{Pub}(p k, C, w)=\operatorname{HPS} \operatorname{Priv}(s k, C)$ (it holds for all $C \in \mathcal{V})$.

The encapsulated key $K$ functions in two ways. (1) It serves as an input, together with a random string $s$, to extractor $\operatorname{Ext}(K, s)$ to mask and hide the plaintext $M$ to deal with key leakage. (2) It serves as the input of $\operatorname{LF}_{F p k, t}(\cdot)$ to check the well-formedness of the ciphertext. Tag $t=\left(t_{a}, t_{c}\right)$ is determined by $t_{a}=(C, s, \Psi)$ and a random $t_{c}$. $\operatorname{LF}_{F p k, t}(K)$ can also be considered as an authentication code, which is used to authenticate the tag $t=\left((C, s, \Psi), t_{c}\right)$ with the authentication key $K$.

In the security proof, some changes are made to the generation of the challenge ciphertext $C T^{*}=\left(C^{*}, s^{*}, \Psi^{*}, \Pi^{*}, t_{c}^{*}\right): C^{*}$ is sampled from $\mathcal{C} \backslash \mathcal{V}$ and the tag $t^{*}$ is made lossy by computing a proper $t_{c}$ with trapdoor Ftd. A PPT adversary cannot tell the changes due to the hardness of subset membership problem and the indistinguishability of lossy tags and injective ones. Conditioned on $C T^{*}$, the encapsulated key $K^{*}=\operatorname{HPS} \cdot \operatorname{Priv}\left(s k, C^{*}\right)$ still maintains a high min-entropy since $\Pi^{*}=\operatorname{LF}_{F p k, t^{*}}\left(K^{*}\right)$ works in lossy mode and only little information is released. When a PPT adversary chooses an invalid ciphertext $C T$ in the sense that $C \in \mathcal{C} \backslash \mathcal{V}$ for decryption query, the corresponding tag $t$ is injective with overwhelming probability. Then $\operatorname{LF}_{F p k, t}(\cdot)$ is injective and $\Pi$ preserves the high min-entropy of $K=$ HPS.Priv $(s k, C)$. Hence invalid ciphertexts will be rejected by the decryption oracle with overwhelming probability. On the other hand, the information of $p k$ has already determined $K=\operatorname{HPS} \cdot \operatorname{Priv}(s k, C)$ for all $C \in$ $\mathcal{V}$. Thus the decryption oracle does not help the adversary to gain any more information about $K^{*}$. Then an extractor can be applied to $K^{*}$ to totally mask the information of challenge plaintext, and a large min-entropy of $K^{*}$ conditioned on $p k$ and $\Pi^{*}$ implies a high tolerance of key leakage.

Thanks to efficient constructions for HPS and OT-LF under the DDH and DCR assumptions, the instantiations are practically efficient. More precisely, $|K| \approx L / 2$, where $L$ is the length of the secret key of HPS. Due to the lossiness of the OT-LF and the property of the HPS, the min-entropy conditioned on the public key and challenge ciphertext, approaches $(1 / 2-o(1)) L$. Hence the leakage rate approaches $1 / 2$.

\section{Preliminaries}

Notation. Let $[n]$ denote the set $\{1, \ldots, n\}$. Let $\kappa \in \mathbb{N}$ denote the security parameter and $1^{\kappa}$ denote the string of $\kappa$ ones. If $s$ is a string, then $|s|$ denotes its length, while if $S$ is a set then $|S|$ denotes its size and $s \leftarrow S$ denotes the operation of picking an element $s$ uniformly at random from $S$. We denote $y \leftarrow A(x)$ the 
operation of running $A$ with input $x$, and assigning $y$ as the result. We write $\log s$ for logarithms over the reals with base 2 .

Randomness Extractor. Let $\operatorname{SD}(X, Y)$ denote the statistical distance of random variables $X$ and $Y$ over domain $\Omega$. Namely, $\operatorname{SD}(X, Y)=\frac{1}{2} \sum_{\omega \in \Omega} \mid \operatorname{Pr}[X=$ $\omega]-\operatorname{Pr}[Y=\omega] \mid$. The min-entropy of $X$ is $\mathrm{H}_{\infty}(X)=-\log \left(\max _{\omega \in \Omega} \operatorname{Pr}[X=\omega]\right)$. Dodis et al. 13 formalized the notion of average min-entropy of $X$ conditioned on $Y$ which is defined as $\widetilde{\mathrm{H}}_{\infty}(X \mid Y)=-\log \left(E_{y \leftarrow Y}\left[2^{-\mathrm{H}_{\infty}(X \mid Y=y)}\right]\right)$. They proved the following property of average min-entropy.

Lemma 1. [13] Let $X, Y$ and $Z$ be random variables. If $Y$ has at most $2^{r}$ possible values, then $\widetilde{\mathrm{H}}_{\infty}(X \mid(Y, Z)) \geq \widetilde{\mathrm{H}}_{\infty}(X \mid Z)-r$.

Definition 1 (Randomness Extractor). An efficient function Ext : $\mathcal{X} \times \mathcal{S} \rightarrow$ $\mathcal{Y}$ is an average-case $(\nu, \epsilon)$-strong extractor if for all pairs of random variables $(X, Z)$ such that $X \in \mathcal{X}$ and $\widetilde{\mathrm{H}}_{\infty}(X \mid Z) \geq \nu$, we have

$$
\operatorname{SD}\left((Z, s, \operatorname{Ext}(X, s)),\left(Z, s, U_{\mathcal{Y}}\right)\right) \leq \epsilon,
$$

where $s$ is uniform over $\mathcal{S}$ and $U_{\mathcal{Y}}$ is uniform over $\mathcal{Y}$.

A family of universal hash functions $\mathcal{H}=\left\{H_{s}: \mathcal{X} \rightarrow \mathcal{Y}\right\}_{s \in \mathcal{S}}$ can be used as an average-case $\left(\widetilde{\mathrm{H}}_{\infty}(X \mid Z), \epsilon\right)$-strong extractors whenever $\widetilde{\mathrm{H}}_{\infty}(X \mid Z) \geq \log |\mathcal{Y}|+$ $2 \log (1 / \epsilon)$, according to the general Leftover Hash Lemma [13.

\subsection{Leakage-Resilient Public-Key Encryption}

A Public-Key Encryption (PKE) scheme with plaintext space $\mathcal{M}$ is given by three PPT algorithms (PKE.Gen, PKE.Enc, PKE.Dec). The key generation algorithm PKE.Gen takes as input $1^{\kappa}$, and outputs a pair of public/secret keys $(P K, S K)$. The encryption algorithm PKE.Enc takes as input a public key $P K$ and a plaintext $M \in \mathcal{M}$, and returns a ciphertext $C T=\operatorname{PKE}$.Enc $(P K, M)$. The decryption algorithm PKE.Dec takes as input a secret key $S K$ and a ciphertext $C T$, and returns a plaintext $M \in \mathcal{M} \cup\{\perp\}$. For consistency, we require that PKE.Dec $(S K$, PKE.Enc $(P K, M))=M$ holds for all $(P K, S K) \leftarrow \operatorname{PKE} . G e n\left(1^{\kappa}\right)$ and all plaintexts $M \in \mathcal{M}$.

Following [27/28, we define leakage-resilient chosen-ciphertext security (LRCCA) for PKE.

Definition 2 (Leakage-Resilient CCA security of PKE). A public-key encryption scheme PKE $=$ (PKE.Gen, PKE.Enc, PKE.Dec) is $\lambda$-leakage-resilient chosen-ciphertext secure $(\lambda$-LR-CCA-secure), if for any PPT adversary $\mathcal{A}=$ $\left(\mathcal{A}_{1}, \mathcal{A}_{2}\right)$, the function $\operatorname{Adv}_{\mathrm{PKE}, \mathcal{A}}^{\text {lr-cca }}(\kappa):=\left|\operatorname{Pr}\left[\operatorname{Exp}_{\mathrm{PKE}, \mathcal{A}}^{\text {lr-cca }}(\kappa)=1\right]-\frac{1}{2}\right|$ is negligible in $\kappa$. Below defines ExpPKE, $\mathcal{A}(\kappa)$.

1. $(P K, S K) \leftarrow$ PKE.Gen $\left(1^{\kappa}\right), b \leftarrow\{0,1\}$.

2. $\left(M_{0}, M_{1}\right.$, state $) \leftarrow \mathcal{A}_{1}^{\mathcal{O}_{s k}^{\lambda, \kappa}(\cdot), \operatorname{PKE} \cdot \operatorname{Dec}(S K, \cdot)}(p k)$, s.t. $\left|M_{0}\right|=\left|M_{1}\right|$.

3. $C T^{*} \leftarrow \operatorname{PKE} . \operatorname{Enc}\left(P K, M_{b}\right) . b^{\prime} \leftarrow \mathcal{A}_{2}^{\mathrm{PKE} \cdot \operatorname{Dec}_{\neq C T^{*}}(S K, \cdot)}\left(\right.$ state,$\left.C T^{*}\right)$.

5. If $b=b^{\prime}$ return 1 else return 0 .

In the case of $\lambda=0$, Definition 2 is just the standard CCA security 32 . 


\subsection{Hash Proof System}

We recall the notion of hash proof systems introduced by Cramer and Shoup [9]. For simplicity, hash proof systems are described as key encapsulation mechanisms (KEMs), as did in 21.

Projective Hashing. Let $\mathcal{S} \mathcal{K}, \mathcal{P} \mathcal{K}$ and $\mathcal{K}$ be sets of public keys, secret keys and encapsulated keys. Let $\mathcal{C}$ be the set of all ciphertexts of KEM and $\mathcal{V} \subset \mathcal{C}$ be the set of all valid ones. We assume that there are efficient algorithms for sampling $s k \leftarrow \mathcal{S K}, C \leftarrow \mathcal{V}$ together with a witness $w$, and $C \leftarrow \mathcal{C} \backslash \mathcal{V}$.

Let $\Lambda_{s k}: \mathcal{C} \rightarrow \mathcal{K}$ be a hash function indexed with $s k \in \mathcal{S K}$ that maps ciphertexts to symmetric keys. The hash function $\Lambda_{s k}$ is projective if there exists a projection $\mu: \mathcal{S K} \rightarrow \mathcal{P K}$ such that $\mu(s k) \in \mathcal{P} \mathcal{K}$ defines the action of $\Lambda_{s k}$ over the subset $\mathcal{V}$ of valid ciphertexts.

Definition 3 (universal[9]). A projective hash function $\Lambda_{s k}$ is $\epsilon$-universal, if for all $p k, C \in \mathcal{C} \backslash \mathcal{V}$, and all $K \in \mathcal{K}$, it holds that $\operatorname{Pr}\left[\Lambda_{s k}(C)=K \mid(p k, C)\right] \leq \epsilon$, where the probability is over all possible $s k \leftarrow \mathcal{S K}$ with $p k=\mu(s k)$.

The lemma below follows directly from the definition of min-entropy.

Lemma 2. Assume that $\Lambda_{s k}: \mathcal{C} \rightarrow \mathcal{K}$ is an $\epsilon$-universal projective hash function. Then, for all $p k$ and $C \in \mathcal{C} \backslash \mathcal{V}$, it holds that $\mathrm{H}_{\infty}\left(\Lambda_{s k}(C) \mid(p k, C)\right) \geq \log 1 / \epsilon$, where $s k \leftarrow \mathcal{S K}$ with $p k=\mu(s k)$.

Hash Proof System. A hash proof system HPS consists of three PPT algorithms (HPS.Gen, HPS.Pub, HPS.Priv). The parameter generation algorithm HPS.Gen $\left(1^{\kappa}\right)$ generates parameterized instances of the form params $=($ group, $\mathcal{K}$, $\left.\mathcal{C}, \mathcal{V}, \mathcal{S} \mathcal{K}, \mathcal{P} \mathcal{K}, \Lambda_{(\cdot)}: \mathcal{C} \rightarrow \mathcal{K}, \mu: \mathcal{S} \mathcal{K} \rightarrow \mathcal{P} \mathcal{K}\right)$, where group may contain additional structural parameters. The public evaluation algorithm HPS.Pub $(p k, C, w)$ takes as input a projective public key $p k=\mu(s k)$, a valid ciphertext $C \in \mathcal{V}$ and a witness $w$ of the fact that $C \in \mathcal{V}$, and computes the encapsulated key $K=\Lambda_{s k}(C)$. The private evaluation algorithm HPS.Priv $(s k, C)$ takes a secret key $s k$ and a ciphertext $C \in \mathcal{V}$ as input, and returns the encapsulated key $K=\Lambda_{s k}(C)$ without knowing a witness. We assume that $\mu$ and $\Lambda_{(\cdot)}$ are efficiently computable.

Subset Membership Problem. The subset membership problem associated with a HPS suggests that a random valid ciphertext $C_{0} \leftarrow \mathcal{V}$ and a random invalid ciphertext $C_{1} \leftarrow \mathcal{C} \backslash \mathcal{V}$ are computationally indistinguishable. This is formally captured by a negligible advantage function $\operatorname{Adv}_{\mathrm{HPS}, \mathcal{A}}^{\mathrm{smp}}(\kappa)$ for all PPT adversary $\mathcal{A}$, where

$$
\operatorname{Adv}_{\mathrm{HPS}, \mathcal{A}}^{\mathrm{smp}}(\kappa)=\left|\operatorname{Pr}\left[\mathcal{A}\left(\mathcal{C}, \mathcal{V}, C_{0}\right)=1 \mid C_{0} \leftarrow \mathcal{V}\right]-\operatorname{Pr}\left[\mathcal{A}\left(\mathcal{C}, \mathcal{V}, C_{1}\right)=1 \mid C_{1} \leftarrow \mathcal{C} \backslash \mathcal{V}\right]\right|
$$

Definition 4. A hash proof system HPS $=$ (HPS.Gen, HPS.Pub, HPS.Priv) is $\epsilon$ universal if: (i) for all sufficiently large $\kappa \in \mathbb{N}$ and for all possible outcomes of HPS.Gen $\left(1^{\kappa}\right)$, the underlying projective hash function is $\epsilon(\kappa)$-universal for negligible $\epsilon(\kappa)$; (ii) the underlying subset membership problem is hard. Furthermore, a hash proof system is called perfectly universal if $\epsilon(\kappa)=1 /|\mathcal{K}|$. 


\subsection{One-Time Lossy Filter}

One-time Lossy Filter (OT-LF) is a simplified version of lossy algebraic filters recently introduced by Hofheinz [19. A (Dom, $\left.\ell_{\mathrm{LF}}\right)$-OT-LF is a family of functions indexed by a public key $F p k$ and a tag $t$. A function $\mathrm{LF}_{F p k, t}$ from the family maps an input $X \in$ Dom to an output $\operatorname{LF}_{F p k, t}(X)$. Given public key $F p k$, the set of tags $\mathcal{T}$ contains two computationally indistinguishable disjoint subsets, namely the subset of injective tags $\mathcal{T}_{\text {inj }}$ and the subset of lossy ones $\mathcal{T}_{\text {loss }}$. If $t$ is an injective tag, the function $\mathrm{LF}_{F p k, t}$ is injective and has image size of $|\mathrm{Dom}|$. If $t$ is lossy, the output of the function has image size at most $2^{\ell_{\mathrm{LF}}}$. Thus, a lossy tag ensures that $\mathrm{LF}_{F p k, t}(X)$ reveals at most $\ell_{\mathrm{LF}}$ bits of information about its input $X$. This is a crucial property of an LF.

Definition 5 (OT-LF). A (Dom, $\ell_{\mathrm{LF}}$ )-one-time lossy filter LF consists of three PPT algorithms (LF.Gen, LF.Eval, LF.LTag):

Key Generation. LF.Gen $\left(1^{\kappa}\right)$ outputs a key pair $(F p k, F t d)$. The public key $F p k$ defines a tag space $\mathcal{T}=\{0,1\}^{*} \times \mathcal{T}_{c}$ that contains two disjoint subsets, the subset of lossy tags $\mathcal{T}_{\text {loss }} \subseteq \mathcal{T}$ and that of injective tags $\mathcal{T}_{\text {inj }} \subseteq \mathcal{T}$. A $\operatorname{tag} t=\left(t_{a}, t_{c}\right) \in \mathcal{T}$ consists of an auxiliary tag $t_{a} \in\{0,1\}^{*}$ and a core tag $t_{c} \in \mathcal{T}_{c}$. Ftd is a trapdoor that allows to efficiently sample a lossy tag.

Evaluation. LF.Eval $(F p k, t, X)$, for a public key $F p k$, a tag $t$ and $X \in$ Dom, computes $\operatorname{LF}_{F p k, t}(X)$.

Lossy Tag Generation. LF.LTag $\left(F t d, t_{a}\right)$, for an auxiliary tag $t_{a}$ and the trapdoor $F t d$, computes a core tag $t_{c}$ such that $t=\left(t_{a}, t_{c}\right)$ is lossy.

We require that an OT-LF LF has the following properties:

Lossiness. If $t$ is injective, so is the function $\operatorname{LF}_{F p k, t}(\cdot)$. If $t$ is lossy, then $\mathrm{LF}_{F p k, t}(X)$ has image size of at most $2^{\ell_{\mathrm{LF}}}$. (In application, we are interested in OT-LFs that have a constant parameter $\ell_{\mathrm{LF}}$ even for larger domain.)

Indistinguishability. For any PPT adversary $\mathcal{A}$, it is hard to distinguish a lossy tag from a random tag, i.e., the following advantage is negligible in $\kappa$.

$$
\operatorname{Adv}_{\mathrm{LF}, \mathcal{A}}^{\mathrm{ind}}(\kappa):=\mid \operatorname{Pr}\left[\mathcal{A}\left(F p k,\left(t_{a}, t_{c}^{(0)}\right)\right)=1\right]-\operatorname{Pr}\left[\mathcal{A}\left(F p k,\left(t_{a}, t_{c}^{(1)}\right)\right)=1 \mid\right.
$$

where $(F p k, F t d) \leftarrow \operatorname{LF} . G e n\left(1^{\kappa}\right), t_{a} \leftarrow \mathcal{A}(F p k), t_{c}^{(0)} \leftarrow \operatorname{LF} . \operatorname{LTag}\left(F t d, t_{a}\right)$ and $t_{c}^{(1)} \leftarrow \mathcal{T}_{c}$.

Evasiveness. For any PPT adversary $\mathcal{A}$, it is hard to generate a non-injective tag 1 even given a lossy tag, i.e., the following advantage is negligible in $\kappa$.



Remark 1. The definition of one-time lossy filter is different from that of lossy algebraic filter [19] in two ways. First, the one-time property in our definition allows the adversary to query lossy tag generation oracle only once in both indistinguishability and evasiveness games. While in [19, the adversary is allowed to query the oracle polynomial times. Secondly, unlike lossy algebraic filter, onetime lossy filter does not require any algebraic properties.

\footnotetext{
${ }^{1}$ In some case, a tag may neither injective nor lossy.
} 


\subsection{Chameleon Hashing}

A chameleon hashing function [22] is essentially a hashing function associated with a pair of evaluation key and trapdoor. Its collision-resistant property holds when only the evaluation key of the function is known, but is broken with the trapdoor. We recall the formal definition of chameleon hashing from [18].

Definition 6 (Chameleon Hashing). A chameleon hashing function $\mathrm{CH}$ consists of three PPT algorithms (CH.Gen, CH.Eval, CH.Equiv):

Key Generation. CH.Gen $\left(1^{\kappa}\right)$ outputs an evaluation key $e k_{\mathrm{CH}}$ and a trapdoor $t d_{\mathrm{CH}}$.

Evaluation. CH.Eval $\left(e k_{\mathrm{CH}}, x ; r_{\mathrm{CH}}\right)$ maps $x \in\{0,1\}^{*}$ to $y \in \mathcal{Y}$ with help of the evaluation key $e k_{\mathrm{CH}}$ and a randomness $r_{\mathrm{CH}} \leftarrow \mathcal{R}_{\mathrm{CH}}$. If $r_{\mathrm{CH}}$ is uniformly distributed over $\mathcal{R}_{\mathrm{CH}}$, so is $y$ over $\mathcal{Y}$.

Equivocation. CH.Equiv $\left(t d_{\mathrm{CH}}, x, r_{\mathrm{CH}}, x^{\prime}\right)$ outputs a randomness $r_{\mathrm{CH}}^{\prime} \in \mathcal{R}_{\mathrm{CH}}$ such that

$$
\mathrm{CH} . \mathrm{Eval}\left(e k_{\mathrm{CH}}, x ; r_{\mathrm{CH}}\right)=\mathrm{CH} . \mathrm{Eval}\left(e k_{\mathrm{CH}}, x^{\prime} ; r_{\mathrm{CH}}^{\prime}\right) \text {, }
$$

for all $x, x^{\prime}$ and $r_{\mathrm{CH}}$. Meanwhile, $r_{\mathrm{CH}}^{\prime}$ is uniformly distributed as long as $r_{\mathrm{CH}}$ is.

Collision Resistance. Given evaluation key $e k_{\mathrm{CH}}$, it is hard to find $\left(x, r_{\mathrm{CH}}\right) \neq$ $\left(x^{\prime}, r_{\mathrm{CH}}^{\prime}\right)$ with CH.Eval $\left(e k_{\mathrm{CH}}, x ; r_{\mathrm{CH}}\right)=\mathrm{CH} . \mathrm{Eval}\left(e k_{\mathrm{CH}}, x^{\prime} ; r_{\mathrm{CH}}^{\prime}\right)$. More precisely, for any PPT adversary $\mathcal{A}$, the following advantage is negligible in $\kappa$.

$$
\operatorname{Adv}_{\mathrm{CH}, \mathcal{A}}^{\mathrm{cr}}(\kappa):=\operatorname{Pr}\left[\begin{array}{c}
\left(x, r_{\mathrm{CH}}\right) \neq\left(x^{\prime}, r_{\mathrm{CH}}^{\prime}\right) \\
\wedge E q . \text { (11)holds. }
\end{array}: \begin{array}{l}
\left(e k_{\mathrm{CH}}, t d_{\mathrm{CH}}\right) \leftarrow \mathrm{CH} \cdot \mathrm{Gen}\left(1^{\kappa}\right) \\
\left(x, r_{\mathrm{CH}}, x^{\prime}, r_{\mathrm{CH}}^{\prime}\right) \leftarrow \mathcal{A}\left(e k_{\mathrm{CH}}\right)
\end{array}\right]
$$

\section{The Construction}

Let HPS = (HPS.Gen, HPS.Pub, HPS.Priv) be an $\epsilon_{1}$-universal hash proof system, where HPS.Gen $\left(1^{\kappa}\right)$ generates instances of params $=($ group $, \mathcal{K}, \mathcal{C}, \mathcal{V}, \mathcal{S K}, \mathcal{P} \mathcal{K}$, $\left.\Lambda_{(\cdot)}: \mathcal{C} \rightarrow \mathcal{K}, \mu: \mathcal{S K} \rightarrow \mathcal{P K}\right)$. Let LF $=\left(\right.$ LF.Gen, LF.Eval, LF.LTag) be a $\left(\mathcal{K}, \ell_{\mathrm{LF}}\right)-$ one-time lossy filter. Define $\nu:=\log \left(1 / \epsilon_{1}\right)$. Let $\lambda$ be a bound on the amount of leakage, and let Ext : $\mathcal{K} \times\{0,1\}^{d} \rightarrow\{0,1\}^{m}$ be an average-case $\left(\nu-\lambda-\ell_{\mathrm{LF}}, \epsilon_{2}\right)$ strong extractor. We assume that $\epsilon_{2}$ is negligible in $\kappa$. The encryption scheme PKE $=\left(\right.$ PKE.Gen, PKE.Enc, PKE.Dec) with plaintext space $\{0,1\}^{m}$ is described as follows.

Key Generation. PKE.Gen $\left(1^{\kappa}\right)$ runs HPS.Gen $\left(1^{\kappa}\right)$ to obtain params and runs LF.Gen $\left(1^{\kappa}\right)$ to obtain $(F p k, F t d)$. It also picks $s k \leftarrow \mathcal{S K}$ and sets $p k=$ $\mu(s k)$. The output is a public/secret key pair $(P K, S K)$, where $P K=$ (params, $F p k, p k$ ) and $S K=s k$.

Encryption. PKE. Enc $(P K, M)$ takes as input a public key $P K$ and a message $M \in\{0,1\}^{m}$. It chooses $C \leftarrow \mathcal{V}$ with witness $w$, a random seed $s \leftarrow\{0,1\}^{d}$ and a random core tag $t_{c} \leftarrow \mathcal{T}_{c}$. It then computes

$$
K=\operatorname{HPS} \cdot \operatorname{Pub}(p k, C, w), \Psi=\operatorname{Ext}(K, s) \oplus M, \Pi=\operatorname{LF}_{F p k, t}(K),
$$

where the filter tag is $t=\left(t_{a}, t_{c}\right)$ with $t_{a}=(C, s, \Psi)$. Output the ciphertext $C T=\left(C, s, \Psi, \Pi, t_{c}\right)$. 
Decryption. PKE.Dec $(S K, C T)$, given a secret key $S K=s k$ and a ciphertext $C T=\left(C, s, \Psi, \Pi, t_{c}\right)$, computes $K^{\prime}=\operatorname{HPS} \cdot \operatorname{Priv}(s k, C)$ and $\Pi^{\prime}=\mathrm{LF}_{F p k, t}\left(K^{\prime}\right)$, where $t=\left((C, s, \Psi), t_{c}\right)$. It checks whether $\Pi=\Pi^{\prime}$. If not, it rejects with $\perp$. Otherwise it outputs $M=\Psi \oplus \operatorname{Ext}\left(K^{\prime}, s\right)$.

The correctness of PKE follows from the correctness of the underlying hash proof system.

The idea of our construction is to employ a Hash Proof System (HPS) to generate an encapsulated key $K$, which is then used not only to mask the plaintext, but also to verify the well-formedness of the ciphertext. To deal with the secret key leakage, an extractor converts $K$ to a shorter key to hide the plaintext $M$. A one-time lossy filter $\mathrm{LF}_{F p k, t}(K)$ helps to implement the verification. The filter in the challenge ciphertext $C T^{*}$ works in the lossy mode, and it leaks only a limited amount of information about the key $K$. For any invalid ciphertext submitted by the adversary to the decryption oracle, the filter works in the injective mode with overwhelming probability. Consequently, the output of the filter in the invalid ciphertext preserves the entropy of $K$, which makes the ciphertext rejected by the decryption oracle with overwhelming probability.

The security of the construction is established by the theorem below.

Theorem 1. Assuming that HPS is an $\epsilon_{1}$-universal hash proof system, LF is a $\left(\mathcal{K}, \ell_{\mathrm{LF}}\right)$-one-time lossy filter, and $\mathrm{Ext}: \mathcal{K} \times\{0,1\}^{d} \rightarrow\{0,1\}^{m}$ is an average-case $\left(\nu-\lambda-\ell_{\mathrm{LF}}, \epsilon_{2}\right)$-strong extractor, the encryption scheme PKE is $\lambda$-LR-CCAsecure as long as $\lambda \leq \nu-m-\ell_{\mathrm{LF}}-\omega(\log \kappa)$, where $m$ is the plaintext length and $\nu:=\log \left(1 / \epsilon_{1}\right)$. Particularly,

$\operatorname{Adv}_{\mathrm{PKE}, \mathcal{A}}^{\mathrm{Ir}-\mathrm{cca}}(\kappa) \leq \operatorname{Adv}_{\mathrm{LF}, \mathcal{B}_{1}}^{\mathrm{ind}}(\kappa)+Q(\kappa) \cdot \operatorname{Adv}_{\mathrm{LF}, \mathcal{B}_{2}}^{\text {eva }}(\kappa)+\operatorname{Adv}_{\mathrm{HPS}, \mathcal{B}_{3}}^{\operatorname{smp}}(\kappa)+\frac{Q(\kappa) 2^{\lambda+\ell_{\mathrm{LF}}+m}}{2^{\nu}-Q(\kappa)}+\epsilon_{2}$

where $Q(\kappa)$ denotes the number of decryption queries made by $\mathcal{A}$.

Parameters and Leakage Rate. To make our construction tolerate leakage as much as possible, it is useful to consider a "very strong" hash proof system (i.e., $\left.\epsilon_{1} \leq 2 /|\mathcal{K}|\right)$. In this case, $\nu=\log \left(1 / \epsilon_{1}\right) \geq \log |\mathcal{K}|-1$. Thus, when $\mathcal{K}$ is sufficiently large, the leakage rate (defined as $\lambda /|S K|$ ) in our construction approaches $(\log |\mathcal{K}|) /|S K|$ asymptotically.

CCA-Security. Clearly, if $\lambda=0$ and $\log \left(1 / \epsilon_{1}\right) \geq m+\ell_{\mathrm{LF}}+\omega(\log \kappa)$, the above construction is CCA-secure. Thus, it provides a new approach for constructing CCA-secure PKE from any universal hash proof system and OT-LF.

Proof. The proof goes with game arguments [33. We define a sequence of games, $\mathrm{Game}_{0}, \ldots, \mathrm{Game}_{6}$, played between a simulator Sim and a PPT adversary $\mathcal{A}$. In each game, the adversary outputs a bit $b^{\prime}$ as a guess of the random bit $b$ used by the simulator. Denote by $S_{i}$ the event that $b=b^{\prime}$ in $\mathrm{Game}_{i}$ and denote by $C T^{*}=\left(C^{*}, s^{*}, \Psi^{*}, \Pi^{*}, t_{c}^{*}\right)$ the challenge ciphertext.

$\mathrm{Game}_{0}$ : This is the original LR-CCA game $\operatorname{Exp}_{\mathrm{PKE}, \mathcal{A}}^{\mathrm{Ir}-\mathrm{Aca}}(\kappa)$. The simulator generates the public/secret key pair $(P K, S K)$ by invoking PKE.Gen $\left(1^{\kappa}\right)$ and sends the 
public key $P K$ to the adversary $\mathcal{A}$. For each decryption query $C T$ or leakage query $f_{i}$, Sim responds with PKE.Dec $(S K, C T)$ or $f_{i}(S K)$ using secret key $S K$. Upon receiving two messages $M_{0}, M_{1}$ of equal length from the adversary, Sim selects a random $b \in\{0,1\}$ and sends the challenge ciphertext $C T^{*}:=$ PKE.Enc $\left(P K, M_{b}\right)$ to $\mathcal{A}$. The simulator continues to answer the adversary's decryption query as long as $C T \neq C T^{*}$. Finally, $\mathcal{A}$ outputs a bit $b^{\prime}$, which is a guess of $b$. By the Definition 22, we have $\operatorname{Adv}_{\mathrm{PKE}, \mathcal{A}}^{\text {lr-cca }}(\kappa):=\left|\operatorname{Pr}\left[S_{0}\right]-\frac{1}{2}\right|$.


eration of the core $\operatorname{tag} t_{c}^{*}$ of the filter tag in the challenge ciphertext. When calling PKE.Gen $\left(1^{\kappa}\right)$, the simulator keeps the trapdoor Ftd of LF as well as $S K$. Instead of sampling $t_{c}^{*}$ at random from $\mathcal{T}_{c}$, Sim computes $t_{c}^{*}$ with $\operatorname{LF} . \operatorname{LTag}\left(F t d, t_{a}^{*}\right)$, where $t_{a}^{*}=\left(C^{*}, s^{*}, \Psi^{*}\right)$. A straightforward reduction to LF's indistinguishability of lossy tag and random tag yields $\left|\operatorname{Pr}\left[S_{1}\right]-\operatorname{Pr}\left[S_{0}\right]\right| \leq$ $\operatorname{Adv}_{\mathrm{LF}, \mathcal{B}_{1}}^{\text {ind }}(\kappa)$ for a suitable adversary $\mathcal{B}_{1}$ on LF's indistinguishability.

Game $_{2}$ : This game is exactly like $G_{a m e}$, except that a special rejection rule applies to the decryption oracle. If the adversary queries a ciphertext $C T=$ $\left(C, s, \Psi, \Pi, t_{c}\right)$ such that $t=\left(t_{a}, t_{c}\right)=\left(t_{a}^{*}, t_{c}^{*}\right)=t^{*}$, then the decryption oracle immediately outputs $\perp$ and halts. For convenient, we call such tag a copied LF tag. We show that a decryption query with a copied LF tag is

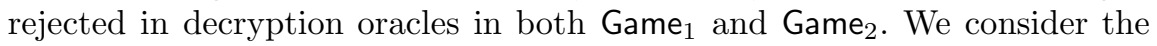
following two cases.

- case 1: $\Pi=\Pi^{*}$. This implies $C T=C T^{*}$. In this case the decryption oracles in Game 1 and $G_{a m e}$ proceed identically since $\mathcal{A}$ is not allowed to ask for the decryption of challenge ciphertext.

- case 2: $\Pi \neq \Pi^{*}$. Since $t=\left((C, s, \Psi), t_{c}\right)=\left(\left(C^{*}, s^{*}, \Psi^{*}\right), t_{c}^{*}\right)=t^{*}$, it follows that $K=K^{*}$, and thus $\operatorname{LF}_{F p k, t}(K)=\operatorname{LF}_{F p k, t^{*}}\left(K^{*}\right)=\Pi^{*}$. So, such decryption queries would have been rejected already in Game . $_{\text {. }}$

According to above analysis, we have $\operatorname{Pr}\left[S_{2}\right]=\operatorname{Pr}\left[S_{1}\right]$.

Game $_{3}$ : This game is exactly like $\mathrm{Game}_{2}$, except for the generation of $K^{*}$ used in the challenge ciphertext. In this game, Sim computes $K^{*}$ with HPS.Priv $\left(s k, C^{*}\right)$ instead of HPS.Pub $\left(p k, C^{*}, w^{*}\right)$. Since HPS is projective, this change is purely conceptual, and thus $\operatorname{Pr}\left[S_{3}\right]=\operatorname{Pr}\left[S_{2}\right]$.

Game $_{4}$ : This game is exactly like Game 3 , except for the generation of $C^{*}$ in the challenge ciphertext $C T^{*}=\left(C^{*}, s^{*}, \Psi^{*}, \Pi^{*}, t_{c}^{*}\right)$. Now Sim samples $C^{*}$ from $\mathcal{C} \backslash \mathcal{V}$ instead of $\mathcal{V}$. A straightforward reduction to the indistinguishability of the subset membership problem yields $\left|\operatorname{Pr}\left[S_{4}\right]-\operatorname{Pr}\left[S_{3}\right]\right| \leq \operatorname{Adv}_{\mathrm{HPS}, \mathcal{B}_{3}}^{\mathrm{smp}}(\kappa)$ for a suitable adversary $\mathcal{B}_{3}$.

Game $_{5}$ : This game is the same as $\mathrm{Game}_{4}$, except that another special rejection rule is applied to the decryption oracle. If the adversary queries a ciphertext $C T=\left(C, s, \Psi, \Pi, t_{c}\right)$ for decryption such that $C \in \mathcal{C} \backslash \mathcal{V}$, then the decryption oracle immediately outputs $\perp$. Let $\operatorname{bad}_{C}$ be the event that a ciphertext is rejected in Game $_{5}$ that would not have been rejected under the rules of Game $_{4}$. Then Game 5 and Game Th proced identically until event bad $_{C}$ occurs. We have

$$
\left|\operatorname{Pr}\left[S_{5}\right]-\operatorname{Pr}\left[S_{4}\right]\right| \leq \operatorname{Pr}\left[\operatorname{bad}_{C}\right]
$$


by the difference lemma of [33. We show the following lemma shortly (after the main proof), which guarantees that bad $_{C}$ occurs with a negligible probability.

Lemma 3. Suppose that the adversary $\mathcal{A}$ makes at most $Q(\kappa)$ decryption queries. Then

$$
\operatorname{Pr}\left[\operatorname{bad}_{C}\right] \leq Q(\kappa) \cdot \operatorname{Adv}_{\mathrm{LF}, \mathcal{B}}^{\mathrm{eva}}(\kappa)+\frac{Q(\kappa) 2^{\lambda+\ell_{\mathrm{LF}}+m}}{2^{\nu}-Q(\kappa)}
$$

where $\mathcal{B}$ is a suitable adversary attacking on LF's evasiveness.

Game $_{6}$ : This game is exactly like Game ${ }_{5}$, except for the generation of $\Psi^{*}$ in $C T^{*}$. In this game, Sim chooses $\Psi^{*}$ uniformly at random from $\{0,1\}^{m}$ instead of using $\operatorname{Ext}\left(\Lambda_{s k}\left(C^{*}\right), s^{*}\right) \oplus M_{b}$.

Claim 1. For $C^{*} \leftarrow \mathcal{C} \backslash \mathcal{V}$ if the decryption algorithm rejects all invalid ciphertexts, then the value $\Lambda_{s k}\left(C^{*}\right)$ has average min-entropy at least $\nu-\lambda-$ $\ell_{\mathrm{LF}} \geq \omega(\log \kappa)+m$ given all the other values in $\mathcal{A}$ 's view (denoted by view $\left.{ }_{\mathcal{A}}^{\prime}\right)$.

We prove Claim 1 by directly analyzing the average min-entropy of $\Lambda_{s k}\left(C^{*}\right)$ from the adversary's point of view. Since all invalid ciphertexts are rejected by the decryption oracle in both Game $e_{5}$ and $\mathrm{Game}_{6}, \mathcal{A}$ cannot learn more information on the value $\Lambda_{s k}\left(C^{*}\right)$ from the decryption oracle other than $p k$, $C^{*}, \Pi^{*}$ and the key leakage. Recall that $\Pi^{*}$ has only $2^{\ell_{\mathrm{LF}}}$ possible vales and $\mathrm{H}_{\infty}\left(\Lambda_{s k}\left(C^{*}\right) \mid\left(p k, C^{*}\right)\right) \geq \nu$ (which holds for all $p k$ and $C^{*} \in \mathcal{C} \backslash \mathcal{V}$ ). Hence,

$$
\begin{aligned}
\widetilde{\mathrm{H}}_{\infty}\left(\Lambda_{s k}\left(C^{*}\right) \mid \operatorname{view}_{\mathcal{A}}^{\prime}\right) & =\widetilde{\mathrm{H}}_{\infty}\left(\Lambda_{s k}\left(C^{*}\right) \mid p k, C^{*}, \lambda \text {-leakage, } \Pi^{*}\right) \\
& \geq \widetilde{\mathrm{H}}_{\infty}\left(\Lambda_{s k}\left(C^{*}\right) \mid p k, C^{*}\right)-\lambda-\ell_{\mathrm{LF}} \geq \nu-\lambda-\ell_{\mathrm{LF}}
\end{aligned}
$$

according to Lemma 1

Applying an average-case $\left(\nu-\lambda-\ell_{\mathrm{LF}}, \epsilon_{2}\right)$-strong extractor Ext : $\mathcal{K} \times\{0,1\}^{d} \rightarrow$ $\{0,1\}^{m}$ to $\Lambda_{s k}\left(C^{*}\right)$, we have that $\operatorname{Ext}\left(\Lambda_{s k}\left(C^{*}\right), s^{*}\right)$ is $\epsilon_{2}$-close to uniform given $\mathcal{A}$ 's view. Hence,

$$
\left|\operatorname{Pr}\left[S_{6}\right]-\operatorname{Pr}\left[S_{5}\right]\right| \leq \epsilon_{2}
$$

Observe that in $\mathrm{Game}_{6}$, the challenge ciphertext is completely independent of the random coin $b$ picked by the simulator. Thus, $\operatorname{Pr}\left[S_{6}\right]=1 / 2$.

Putting all together, Theorem 1 follows.

It remains to prove Lemma 3. We do it now.

Proof (Proof of Lemma 31). Let $F$ be the event that in $\mathrm{Game}_{4}$ there exists a decryption query $C T=\left(C, s, \Psi, \Pi, t_{c}\right)$, such that $t=\left((C, s, \Psi), t_{c}\right)$ is a noninjective, non-copied tag. We have

$$
\operatorname{Pr}\left[\operatorname{bad}_{C}\right]=\operatorname{Pr}\left[\operatorname{bad}_{C} \wedge F\right]+\operatorname{Pr}\left[\operatorname{bad}_{C} \wedge \bar{F}\right] \leq \operatorname{Pr}[F]+\operatorname{Pr}\left[\operatorname{bad}_{C} \mid \bar{F}\right]
$$

Thus, it suffices to prove the following two claims: Claim 2 and Claim 3 , 
Claim 2. Suppose that the adversary $\mathcal{A}$ makes at most $Q(\kappa)$ decryption queries. If LF is a one-time lossy filter, then

$$
\operatorname{Pr}[F] \leq Q(\kappa) \cdot \operatorname{Adv}_{\mathrm{LF}, \mathcal{B}}^{\mathrm{eva}}(\kappa)
$$

where $\mathcal{B}$ is a suitable adversary on $\mathrm{LF}$ 's evasiveness.

Proof. Given a challenge LF evaluation key $F_{p k}^{*}, \mathcal{B}$ simulates $\mathcal{A}$ 's environment in $\mathrm{Game}_{4}$ as follows. It generates the PKE's public key $P K$ as in Game ${ }_{4}$ but sets $F_{p k}=F_{p k}^{*}$. Note that $\mathcal{B}$ can use PKE's secret key to deal with $\mathcal{A}$ 's decryption queries. To simulate the challenge ciphertext (in which the LF tag should be lossy), $\mathcal{B}$ queries its lossy tag generation oracle once with $t_{a}^{*}=\left(C^{*}, s^{*}, \Psi^{*}\right)$ to proceed $t_{c}^{*}$, where $\left(C^{*}, s^{*}, \Psi^{*}\right)$ are generated as in Game 4 . Finally, $\mathcal{B}$ chooses $i \in$ $[Q(k)]$ uniformly, and outputs the tag $t=\left((C, s, \Psi), t_{c}\right)$ extracted from $\mathcal{A}$ 's $i$-th decryption query $\left(C, s, \Psi, \Pi, t_{c}\right)$. Clearly, if the event $F$ occurs, with probability at least $1 / Q(\kappa), t$ is a non-injective tag. That is $\operatorname{Pr}[F] \leq Q(\kappa) \cdot \operatorname{Adv}_{\mathrm{LF}, \mathcal{B}}^{\text {eva }}(\kappa)$.

Claim 3. Suppose that the adversary $\mathcal{A}$ makes at most $Q(\kappa)$ decryption queries. If HPS is $\epsilon_{1}$-universal, then

$$
\operatorname{Pr}\left[\operatorname{bad}_{C} \mid \bar{F}\right] \leq \frac{Q(\kappa) 2^{\lambda+\ell_{\mathrm{LF}}+m}}{2^{\nu}-Q(\kappa)}
$$

where $\nu=\log \left(1 / \epsilon_{1}\right)$.

Proof. Suppose that $C T=\left(C, s, \Psi, \Pi, t_{c}\right)$ is the first ciphertext that makes bad $_{C}$ happen given $\bar{F}$, i.e. $C \in \mathcal{C} \backslash \mathcal{V}$ but $\Pi=\operatorname{LF}_{F p k, t}\left(\Lambda_{s k}(C)\right)$, where $t=\left((C, s, \Psi), t_{c}\right)$ is an injective LF tag. For simplicity, we call $C T=\left(C, s, \Psi, \Pi, t_{c}\right)$ an invalid ciphertext if $C \in \mathcal{C} \backslash \mathcal{V}$. Denote by view ${ }_{\mathcal{A}}$ the adversary's view prior to submitting the first invalid ciphertext. Observe that only $p k$, the challenge ciphertext $C T^{*}$, and the key leakage of at most $\lambda$ bits reveal information of the secret key to the adversary. According to Lemma 1 , we have

$$
\begin{aligned}
\widetilde{\mathrm{H}}_{\infty}\left(\Lambda_{s k}(C) \mid \operatorname{view}_{\mathcal{A}}\right) & =\widetilde{\mathrm{H}}_{\infty}\left(\Lambda_{s k}(C) \mid p k, C, C T^{*}, \lambda \text {-leakage }\right) \\
& \geq \widetilde{\mathrm{H}}_{\infty}\left(\Lambda_{s k}(C) \mid p k, C, C T^{*}\right)-\lambda \\
& \geq \mathrm{H}_{\infty}\left(\Lambda_{s k}(C) \mid(p k, C)\right)-\lambda-\ell_{\mathrm{LF}}-m \\
& \geq \nu-\lambda-\ell_{\mathrm{LF}}-m
\end{aligned}
$$

Eq. (8) follows from the fact that in the challenge ciphertext $C T^{*}$, only $\Psi^{*}$ and $\Pi^{*}$ are related to the secret key, and $\Psi^{*}$ has at most $2^{m}$ possible values and $\Pi^{*}$ has at most $2^{\ell_{\mathrm{LF}}}$ possible values. Note that the information revealed by $t_{c}^{*}$ has already been completely taken into account by $\Psi^{*}$, since $t_{c}^{*}=$ $\operatorname{LF} . \operatorname{LTag}\left(F t d,\left(C^{*}, s^{*}, \Psi^{*}\right)\right)$ can be regarded as a function of $\Psi^{*}$. Eq. (9) follows from the fact that for all $p k$ and $C \in \mathcal{C} \backslash \mathcal{V}, \mathrm{H}_{\infty}\left(\Lambda_{s k}(C) \mid(p k, C)\right) \geq \log \left(1 / \epsilon_{1}\right)=\nu$ , which is due to the $\epsilon_{1}$-universal property of HPS and Lemma 2, The fact that event $F$ does not occur implies that $t=\left((C, s, \Psi), t_{c}\right)$ is an injective tag. Applying an injective function to a distribution preserves its min-entropy, we 
have $\widetilde{\mathrm{H}}_{\infty}\left(\operatorname{LF}_{F p k, t}\left(\Lambda_{s k}(C)\right) \mid \operatorname{view}_{\mathcal{A}}\right) \geq \nu-\lambda-\ell_{\mathrm{LF}}-m$. Thus, in Game 4 the decryption algorithm accepts the first invalid ciphertext with probability at most $2^{\lambda+\ell_{\mathrm{LF}}+m} / 2^{\nu}$. Observe that the adversary can rule out one more value of $K$ from each rejection of invalid ciphertext. So, the decryption algorithm accepts the $i$-th invalid ciphertext with probability at most $2^{\lambda+\ell_{\mathrm{LF}}+m} /\left(2^{\nu}-i+1\right)$. Since $\mathcal{A}$ makes at most $Q(\kappa)$ decryption queries, it follows that

$$
\operatorname{Pr}\left[\operatorname{bad}_{C} \mid \bar{F}\right] \leq \frac{Q(\kappa) 2^{\lambda+\ell_{\mathrm{LF}}+m}}{2^{\nu}-Q(\kappa)}
$$

which is negligible in $\kappa$ if $\lambda \leq \nu-m-\ell_{\mathrm{LF}}-\omega(\log \kappa)$.

This completes the proof of Lemma 3 .

\section{Instantiation from the DDH Assumption}

This section is organized as follows. In Section 4.1 we present a variant of hash proof system from the Decisional Diffie-Hellman (DDH) assumption [9]. In Section 4.2, we introduce an efficient DDH-based OT-LF. In Section 4.3, we apply the construction in Section 3 to the two building blocks and obtain an efficient DDH-based LR-CCA secure PKE scheme, depicted in Fig. 1. In Section 4.4, we show a comparison of our scheme with some existing LR-CCA secure PKE schemes.

The DDH Assumption. We assume a PPT algorithm $\mathcal{G}\left(1^{\kappa}\right)$ that takes as input $1^{\kappa}$ and outputs a tuple of $\mathbb{G}=\langle q, G, g\rangle$, where $G$ is a cyclic group of prime order $q$ and $g$ is a generator of $G$. The Decisional Diffie-Hellman (DDH) assumption holds iff

$$
\operatorname{Adv}_{G, \mathcal{D}}^{\mathrm{ddh}}(\kappa)=\left|\operatorname{Pr}\left[\mathcal{D}\left(g_{1}, g_{2}, g_{1}^{r}, g_{2}^{r}\right)=1\right]-\operatorname{Pr}\left[\mathcal{D}\left(g_{1}, g_{2}, g_{1}^{r}, g_{2}^{r^{\prime}}\right)=1\right]\right|
$$

is negligible in $\kappa$ for any PPT adversary $\mathcal{D}$, where $g_{1}, g_{2} \leftarrow G, r \leftarrow \mathbb{Z}_{q}$ and $r^{\prime} \leftarrow \mathbb{Z}_{q} \backslash\{r\}$

\subsection{A DDH-Based HPS}

Let $\langle q, G, g\rangle \leftarrow \mathcal{G}\left(1^{\kappa}\right)$ and let $g_{1}, g_{2}$ be two random generators of $G$. Choose $\mathfrak{n} \in \mathbb{N}$. We assume there is an efficient injective mapping $\operatorname{lnj}: G \rightarrow \mathbb{Z}_{2}$. For any $u=\left(u_{1}, \ldots, u_{\mathfrak{n}}\right) \in G^{\mathfrak{n}}$, let $\widetilde{\operatorname{Inj}}(u)=\left(\operatorname{Inj}\left(u_{1}\right), \ldots, \operatorname{lnj}\left(u_{\mathfrak{n}}\right)\right) \in \mathbb{Z}_{q}^{\mathfrak{n}}$. Clearly, $\widetilde{\operatorname{Inj}}$ is also an injection. We define a hash proof system $\mathrm{HPS}_{1}=$ $\left(\mathrm{HPS}_{1}\right.$. Gen, $\mathrm{HPS}_{1}$.Pub, $\mathrm{HPS}_{1}$. Priv) below.

The parameter params $=\left(\right.$ group $\left., \mathcal{K}, \mathcal{C}, \mathcal{V}, \mathcal{S K}, \mathcal{P} \mathcal{K}, \Lambda_{s k}, \mu\right)$ is set up as follows.

\footnotetext{
${ }^{2}$ For example, $G$ is a $q$-order elliptic curve group over finite field $\mathbb{F}_{p}$. For 80 -bit security, $p$ and $q$ can be chosen to be 160-bit primes. In such a group, elements (i.e., elliptic curve points) can be represented by 160 -bit strings.
} 
- group $=\left\langle q, G, g_{1}, g_{2}, \mathfrak{n}\right\rangle, \mathcal{C}=G \times G, \quad \mathcal{V}=\left\{\left(g_{1}^{r}, g_{2}^{r}\right): r \in \mathbb{Z}_{q}\right\}$ with witness set $W=\mathbb{Z}_{q}$.

$-\mathcal{K}=\mathbb{Z}_{q}^{\mathfrak{n}}, \mathcal{S K}=\left(\mathbb{Z}_{q} \times \mathbb{Z}_{q}\right)^{\mathfrak{n}}, \quad \mathcal{P} \mathcal{K}=G^{\mathfrak{n}}$.

- For $s k=\left(x_{i, 1}, x_{i, 2}\right)_{i \in[\mathfrak{n}]} \in \mathcal{S K}$, define $p k=\left(p k_{i}\right)_{i \in[\mathfrak{n}]}=\mu(s k)=\left(g_{1}^{x_{i, 1}} g_{2}^{x_{i, 2}}\right)_{i \in[\mathfrak{n}]}$.

- For all $C=\left(u_{1}, u_{2}\right) \in \mathcal{C}$, define $\Lambda_{s k}(C)=\widetilde{\operatorname{lnj}}\left(\left(u_{1}^{x_{i, 1}} u_{2}^{x_{i, 2}}\right)_{i \in[\mathfrak{n}]}\right)$.

The public evaluation and private evaluation algorithms are defined as follows:

- For all $C=\left(g_{1}^{r}, g_{2}^{r}\right) \in \mathcal{V}$ with witness $r \in \mathbb{Z}_{q}$, define $\operatorname{HPS}_{1} \cdot \operatorname{Pub}(p k, C, r)=$ $\widetilde{\operatorname{Inj}}\left(p k_{1}^{r}, \ldots, p k_{\mathfrak{n}}^{r}\right)$.

- For all $C=\left(u_{1}, u_{2}\right) \in \mathcal{C}$, define $\operatorname{HPS}_{1} \cdot \operatorname{Priv}(s k, C)=\Lambda_{s k}(C)$.

Correctness of $\mathrm{HPS}_{1}$ follows directly by the definitions of $\mu$ and $\Lambda_{s k}$. The subset membership problem in $\mathrm{HPS}_{1}$ is hard because of the DDH assumption. If $\mathfrak{n}=1$, this is just the DDH-based hash proof system introduced by Cramer and Shoup with encapsulated key set $\mathcal{K}=\mathbb{Z}_{q}$, and is known to be perfectly universal [921]. We have the following theorem with proof in the full version of the paper.

Theorem 2. For any $\mathfrak{n} \in \mathbb{N}, \mathrm{HPS}_{1}$ is perfectly universal under the DDH assumption with encapsulated key size $|\mathcal{K}|=q^{\mathfrak{n}}$.

\subsection{A DDH-Based OT-LF}

We use the following notations. If $A=\left(A_{i, j}\right)$ is an $n \times n$ matrix over $\mathbb{Z}_{\widetilde{q}}$, and $\widetilde{g}$ is an element of $\widetilde{q}$-order group $\widetilde{G}$. Then $\widetilde{g}^{A}$ denotes the $n \times n$ matrix $\left(\widetilde{g}^{A_{i, j}}\right)$ over $\widetilde{G}$. Given a vector $X=\left(X_{1}, \ldots, X_{n}\right) \in \mathbb{Z}_{\widetilde{q}}^{n}$ and an $n \times n$ matrix $E=\left(E_{i, j}\right) \in \widetilde{G}^{n \times n}$, define

$$
X \cdot E:=\left(\prod_{i=1}^{n} E_{i, 1}^{X_{i}}, \ldots, \prod_{i=1}^{n} E_{i, n}^{X_{i}}\right) \in \widetilde{G}^{n} .
$$

Let $\mathrm{CH}=$ ( $\mathrm{CH} . \mathrm{Gen}, \mathrm{CH}$.Eval, $\mathrm{CH}$.Equiv) define a chameleon hashing function with image set $\mathbb{Z}_{\widetilde{q}}$. The OT-LF is $L_{1}=\left(\mathrm{LF}_{1}\right.$.Gen, LF .Eval, LF $_{1} \cdot \mathrm{LT}$ ag $)$, as shown below.

Key Generation. $\mathrm{LF}_{1} \cdot \mathrm{Gen}\left(1^{\kappa}\right)$ runs $\mathcal{G}\left(1^{\kappa}\right)$ to obtain $\widetilde{\mathbb{G}}=\langle\widetilde{q}, \widetilde{G}, \widetilde{g}\rangle$ and runs CH.Gen $\left(1^{\kappa}\right)$ to obtain $\left(e k_{\mathrm{CH}}, t d_{\mathrm{CH}}\right)$. Pick a random pair $\left(t_{a}^{*}, t_{c}^{*}\right) \leftarrow\{0,1\}^{*} \times \mathcal{R}_{\mathrm{CH}}$ and compute $b^{*}=\mathrm{CH}$.Eval $\left(e k_{\mathrm{CH}}, t_{a}^{*} ; t_{c}^{*}\right)$. Choose $r_{1}, \ldots, r_{n}, s_{1}, \ldots, s_{n} \leftarrow \mathbb{Z}_{\widetilde{q}}$, and compute an $n \times n$ matrix $A=\left(A_{i, j}\right) \in \mathbb{Z}_{\widetilde{q}}^{n \times n}$ with $A_{i, j}=r_{i} s_{j}$ for $i, j \in[n]$. Compute matrix $E=\widetilde{g}^{A-b^{*} \mathbf{I}} \in \widetilde{G}^{n \times n}$, where $\mathbf{I}$ is the $n \times n$ identity matrix over $\mathbb{Z}_{\widetilde{q}}$. Finally, output $F p k=\left(\widetilde{q}, \widetilde{G}, \widetilde{g}, e k_{\mathrm{CH}}, E\right)$ and $F t d=$ $\left(t d_{\mathrm{CH}}, t_{a}^{*}, t_{c}^{*}\right)$. The tag space is defined as $\mathcal{T}=\{0,1\}^{*} \times \mathcal{R}_{\mathrm{CH}}$, where $\mathcal{T}_{\text {loss }}=$ $\left\{\left(t_{a}, t_{c}\right):\left(t_{a}, t_{c}\right) \in \mathcal{T} \wedge \mathrm{CH}\right.$.Eval $\left.\left(e k_{\mathrm{CH}}, t_{a} ; t_{c}\right)=b^{*}\right\}$ and $\mathcal{T}_{i n j}=\left\{\left(t_{a}, t_{c}\right):\right.$ $\left.\left(t_{a}, t_{c}\right) \in \mathcal{T} \wedge \mathrm{CH} . \mathrm{Eval}\left(e k_{\mathrm{CH}}, t_{a} ; t_{c}\right) \notin\left\{b^{*}, b^{*}-\operatorname{Tr}(A)\right\}\right\}$.

Evaluation. For a tag $t=\left(t_{a}, t_{c}\right) \in\{0,1\}^{*} \times \mathcal{R}_{\mathrm{CH}}$ and an input $X=\left(X_{1}, \ldots\right.$, $\left.X_{n}\right) \in \mathbb{Z}_{\widetilde{q}}^{n}, \mathrm{LF}_{1}$. Eval $(F p k, t, X)$ first computes $b=\mathrm{CH} . \mathrm{Eval}\left(e k_{\mathrm{CH}}, t_{a} ; t_{c}\right)$ and outputs

$$
y=X \cdot\left(E \otimes \widetilde{g}^{b \mathbf{I}}\right),
$$

where " $\otimes$ " denotes the operation of entry-wise multiplication. 
Lossy Tag Generation. For an auxiliary tag $t_{a}, \mathrm{LF}_{1} \cdot \mathrm{LTag}\left(F t d, t_{a}\right)$ computes a core tag $t_{c}=\mathrm{CH}$.Equiv $\left(t d_{\mathrm{CH}}, t_{a}^{*}, t_{c}^{*}, t_{a}\right)$ with the trapdoor $F t d=\left(t d_{\mathrm{CH}}, t_{a}^{*}, t_{c}^{*}\right)$.

Theorem 3. $\mathrm{LF}_{1}$ is a $\left(\mathbb{Z}_{\widetilde{q}}^{n}, \log \widetilde{q}\right)$-OT-LF under the DDH assumption.

Proof. The proof of Theorem 3 is given in the full version of the paper.

\subsection{The DDH-Based PKE Scheme}

Let $\mathbb{G}=\langle q, G, g\rangle$ and $\widetilde{\mathbb{G}}=\langle\widetilde{q}, \widetilde{G}, \widetilde{g}\rangle$ be two group descriptions. Suppose $\mathfrak{n} \in$ $\mathbb{N}$ satisfies $\mathfrak{n} \log q \geq \log \widetilde{q}+\lambda+m+\omega(\log \kappa)$. Set $n=\lceil\mathfrak{n} \log q / \log \widetilde{q}\rceil$. Let $\left(e k_{\mathrm{CH}}, t d_{\mathrm{CH}}\right) \leftarrow \mathrm{CH} . \mathrm{Gen}\left(1^{\kappa}\right)$ be a chameleon hash function with image set $\mathbb{Z}_{\widetilde{q}}$. Let Ext : $\mathbb{Z}_{q}^{\mathfrak{n}} \times\{0,1\}^{d} \rightarrow\{0,1\}^{m}$ be an average-case $\left(\mathfrak{n} \log q-\log \widetilde{q}-\lambda, \epsilon_{2}\right)$-strong extractor. Applying the general construction in Section 3 to the aforementioned DDH-based HPS and OT-LF, we obtain a DDH-based PKE scheme in Fig. 1.

Key Generation. PKE ${ }_{1}$.Gen $\left(1^{\kappa}\right)$ : Choose $g_{1}, g_{2} \leftarrow G$ and $\left(x_{i, 1}, x_{i, 2}\right) \leftarrow \mathbb{Z}_{q}$ for $i \in[\mathfrak{n}]$. Set $p k_{i}=g_{1}^{x_{i, 1}} g_{2}^{x_{i, 2}}$ for $i \in[\mathfrak{n}]$. Also choose a random pair $\left(t_{a}^{*}, t_{c}^{*}\right) \in\{0,1\}^{*} \times \mathcal{R}_{\mathrm{CH}}$ and set $b^{*}=\mathrm{CH}$.Eval $\left(e k_{\mathrm{CH}}, t_{a}^{*} ; t_{c}^{*}\right)$. Choose $r_{1}, \ldots, r_{n}, s_{1}, \ldots, s_{n} \leftarrow \mathbb{Z}_{\widetilde{q}}$, and compute matrix $E=\left(E_{i, j}\right)_{i, j \in[n]} \in \widetilde{\mathbb{G}}^{n \times n}$, where $E_{i, j}=\widetilde{g}^{r_{i} s_{j}}$ for $i, j \in[n], i \neq j$, and $E_{i, i}=\widetilde{g}^{r_{i} s_{i}} \widetilde{g}^{-b^{*}}$ for $i \in[n]$. Return $P K=\left(q, G, g_{1}, g_{2}, \mathfrak{n},\left(p k_{i}\right)_{i \in[\mathfrak{n}]}, \widetilde{q}, \widetilde{G}, \widetilde{g}, E, e k_{\mathrm{CH}}\right)$ and $S K=\left(x_{i, 1}, x_{i, 2}\right)_{i \in[\mathfrak{n}]}$.

Encryption. $\mathrm{PKE}_{1} . \operatorname{Enc}(P K, M)$ : For a public key $P K$ and a message $M \in\{0,1\}^{m}$, it chooses $r \leftarrow \mathbb{Z}_{q}$ and $s \leftarrow\{0,1\}^{d}$. Compute

$$
C=\left(g_{1}^{r}, g_{2}^{r}\right), \quad K=\widetilde{\operatorname{Inj}}\left(p k_{1}^{r}, \ldots, p k_{\mathfrak{n}}^{r}\right), \quad \Psi=\operatorname{Ext}(K, s) \oplus M, \quad \Pi=K \cdot\left(E \otimes \widetilde{g}^{b \mathbf{I}}\right)
$$

where $b=\mathrm{CH}$.Eval $\left(e k_{\mathrm{CH}}, t_{a} ; t_{c}\right)$ for the auxiliary tag $t_{a}=(C, s, \Psi)$ and a random filter core tag $t_{c} \in \mathcal{R}_{\mathrm{CH}}$. Note that in the computation of $\Pi, K$ is regarded as a vector of dimension $n$ over $\mathbb{Z}_{\widetilde{q}}$ (this works well since $\mathfrak{n} \log q \leq n \log \widetilde{q}$ ). Return $C T=\left(C, s, \Psi, \Pi, t_{c}\right) \in G^{2} \times\{0,1\}^{d} \times\{0,1\}^{m} \times \widetilde{G}^{n} \times \mathcal{R}_{\mathrm{CH}}$.

Decryption. $\mathrm{PKE}_{1} \cdot \operatorname{Dec}(S K, C T)$ : For a ciphertext $C T=\left(C, s, \Psi, \Pi, t_{c}\right)$, it parses $C$ as $\left(u_{1}, u_{2}\right) \in G^{2}$ and then computes $K^{\prime}=\widetilde{\operatorname{Inj}}\left(u_{1}^{x_{1,1}} u_{2}^{x_{1,2}}, \ldots, u_{1}^{x_{\mathfrak{n}, 1}} u_{2}^{x_{\mathfrak{n}, 2}}\right)$ and $\Pi^{\prime}=K^{\prime} \cdot\left(E \otimes \widetilde{g}^{b \mathbf{I}}\right)$, where $b=\mathrm{CH} . \operatorname{Eval}\left(e k_{\mathrm{CH}},(C, s, \Psi) ; t_{c}\right)$. Finally, it checks whether $\Pi=\Pi^{\prime}$. If not, it rejects with $\perp$. Else, it returns $M=\Psi \oplus \operatorname{Ext}\left(K^{\prime}, s\right)$.

Fig. 1. A DDH-based PKE Scheme $\mathrm{PKE}_{1}=\left(\mathrm{PKE}_{1}\right.$.Gen, $\mathrm{PKE}_{1}$.Enc, $\mathrm{PKE} \mathrm{E}_{1}$.Dec $)$

Theorem 4. If the DDH assumptions hold in groups $G$ and $\widetilde{G}$, and the $\mathrm{CH}$ is a chameleon hash function, then $\mathrm{PKE}_{1}$ is $\lambda$-LR-CCA secure if $\lambda \leq \mathfrak{n} \log q-\log \widetilde{q}-$ $m-\omega(\log \kappa)$ (i.e., $\mathfrak{n} \geq(\lambda+\log \widetilde{q}+m+\omega(\log \kappa)) / \log q)$. In particular, the leakage rate in $\mathrm{PKE}_{1}$ is $1 / 2-o(1)$ and

$$
\begin{aligned}
\operatorname{Adv}_{\mathrm{PKE}_{1}, \mathcal{A}}^{\operatorname{lr}-\mathrm{cca}}(\kappa) \leq & \operatorname{Adv}_{G, \mathcal{B}_{1}}^{\mathrm{ddh}}(\kappa)+2 \mathfrak{n} \operatorname{Adv}_{\widetilde{G}, \mathcal{B}_{2}}^{\mathrm{ddh}}(\kappa)+\frac{Q(\kappa) \cdot \widetilde{q} \cdot 2^{\lambda+m}}{q^{\mathfrak{n}}-Q(\kappa)}+\epsilon_{2} \\
& +Q(\kappa)\left((2 \mathfrak{n}+1) \operatorname{Adv}_{\widetilde{G}, \mathcal{B}_{2}}^{\mathrm{ddh}}(\kappa)+\operatorname{Adv}_{\mathrm{CH}, \mathcal{B}_{3}}^{\mathrm{cr}}(\kappa)\right)
\end{aligned}
$$

where $Q(\kappa)$ is the number of decryption queries made by $\mathcal{A}$. 
Proof. Theorem 2 showed that the underlying HPS in $\mathrm{PKE}_{1}$ is perfectly universal (i.e., $\left.\epsilon_{1}=1 / q^{\mathfrak{n}}\right)$. Theorem 3 said that the underlying filter is a $\left(\widetilde{q}^{n}, \log \widetilde{q}\right)$-OTLF. Consequently, $\mathrm{PKE}_{1}$ is $\lambda$-LR-CCA secure according to Theorem 1] If the parameter $\mathfrak{n}$ in $\mathrm{PKE}_{1}$ increases, with $\widetilde{q}, m$ fixed, $\lambda /|S K|=(\mathfrak{n} \log q-\log \widetilde{q}-m-$ $\omega(\log \kappa)) /(2 \mathfrak{n} \log q)=1 / 2-o(1)$.

\subsection{Efficiency Discussion}

In this section, we show a comparison of our DDH-based PKE scheme with the existing DDH/DLIN based LR-CCA secure PKE schemes 28 251116] in terms of leakage rate and ciphertext overhead (defined as the difference between the ciphertext length and the embedded message length). Note that the GHV12 scheme is obtained by applying the CHK transformation to the mKDM-sIDCPA secure scheme [16. The GHV12 scheme is LR-CCA secure only if the mKDM-sID-CPA secure scheme [16] is master-key leakage sID-CPA secure. In fact, Galindo et.al. claimed their mKDM-sID-CPA secure scheme is master-key leakage sID-CPA secure with leakage rate $1-o(1)$, but without any rigorous proof. We personally regard that proving that claim is very hard, since the proof involves constructing a PPT simulator to answer not only key leakage queries, but also identities' private key queries. Nevertheless, we include the GHV12 scheme in the comparison. For simplicity, in a ciphertext, we only consider the length of group elements, ignoring the constant length non-group elements, e.g., the seed used in a randomness extractor. We also assume that elements in $q$ order group can be encoded as bit strings of length $\log q$. To be fair, like in [11, Theorem 6], we will consider the ciphertext overhead (shorted as "CT overhead") under any fixed and achievable leakage rate. We begin by giving an overview of the secret key size (shorted as "SK size"), the amount of absolute leakage and the number of group elements in the ciphertexts of the PKE schemes [282516] in Table 1. In table 1, $\kappa$ is the security parameter; $q^{\prime}, q$ and $\widetilde{q}$ are group sizes; $m$ is the message length and $\mathfrak{n}$ is a parameter as in Fig. 1] and [16, Section 5]. In our scheme, $n=\lceil\mathfrak{n} \log q / \log \widetilde{q}\rceil$. So, the bit-length of $n$ elements in group $\widetilde{G}$ equals that of $\mathfrak{n}$ elements in group $G$.

Table 1. Secret-key size, leakage amount and ciphertext overhead

\begin{tabular}{|c|c|c|c|}
\hline Schemes & $\begin{array}{l}\text { SK size } \\
\text { (\# bits) }\end{array}$ & $\begin{array}{l}\text { Leakage amount } \\
\text { (\# bits) }\end{array}$ & $\begin{array}{l}\text { CT overhead } \\
(\# G)\end{array}$ \\
\hline GHV12 [16] & $\mathfrak{n} \log q$ & $\lambda \leq \mathfrak{n} \log q-3 \log q-2 \ell(\kappa)$ & $2 \mathfrak{n}+6$ \\
\hline NS09 28] & $6 \log q^{\prime}$ & $\lambda \leq \log q^{\prime}-\omega(\log \kappa)-m$ & 3 \\
\hline LZSS12 25. & $4 \log q^{\prime}$ & $\lambda \leq \log q^{\prime}-\omega(\log \kappa)-m$ & 3 \\
\hline Ours & $2 \mathfrak{n} \log q$ & $\lambda \leq \mathfrak{n} \log q-\log \widetilde{q}-m-\omega(\log \kappa)$ & $\mathfrak{n}+2$ \\
\hline
\end{tabular}

We observe that in our scheme as well as that of [11]6] the group size (i.e. $q$ and $\widetilde{q}$ ) remains constant even with larger leakage. While in [28] and [25], both of them rely on increasing the group size (i.e., $q^{\prime}$ ) to tolerate larger leakage. So, 
it is more reasonable to compare the bit-length of ciphertext overhead rather than the number of group elements for the same leakage rate. As an example, we give the concrete relations between ciphertext overhead and leakage-rate of our scheme. In our scheme, for a security level $\ell(\kappa)$, we can choose $|q|=|\widetilde{q}|=$ $2 \ell(\kappa)$. From [13, applying a universal hash function to a source with $3 \ell(\kappa)$ entropy suffices to extract $\ell(\kappa)$-bit random key that is $2^{-\ell(\kappa)}$-close to a uniform distribution over $\{0,1\}^{\ell(\kappa)}$. So, we can set $\omega(\log \kappa)=2 \ell(\kappa)$ and $m=\ell(\kappa)$. According to Theorem 4 the amount of leakage is bounded by $(2 \mathfrak{n}-5) \ell(\kappa)$. Thus, for any $\delta \in[0,1 / 2)$, the leakage rate in our scheme achieves $\delta$, as long as $\mathfrak{n} \geq\lceil 5 /(2-4 \delta)\rceil$ (i.e., $\lambda \leq \ell(\kappa)(2\lceil 5 /(2-4 \delta)\rceil-5))$ and the ciphertext overhead is $(\lceil 5 /(2-4 \delta)\rceil+2) 2 \ell(\kappa)$ bits (ignoring the seed and the core tag part).

Similarly, we can compute the other schemes' ciphertext overheads for reasonable leakage rates. We summarize these results in Table 2.

Table 2. Relations between ciphertext overhead and leakage rate

\begin{tabular}{|l|lll|}
\hline Schemes & $\begin{array}{l}\text { CT overhead } \\
(\# \ell(\kappa) \text { bits })\end{array}$ & $\begin{array}{l}\text { Leakage rate Assumption } \\
\text { interval }(\delta)\end{array}$ \\
\hline DHLW10 [1] & $21 /(1-\delta)+70$ & {$[0,1)$} & DLIN (with tSE-NIZK) \\
GHV12 [16] & $4\lceil 4 /(1-\delta)\rceil+12[0,1)$ & DLIN (without proof) \\
NS09 28] & $9 /(1-6 \delta)$ & {$[0,1 / 6)$} & DDH \\
LZSS12 [25] & $9 /(1-4 \delta)$ & {$[0,1 / 4)$} & DDH \\
Ours & $2\lceil 5 /(2-4 \delta)\rceil+4[0,1 / 2)$ & DDH \\
\hline
\end{tabular}

Table 3. Quantitative comparison (\# $\ell(\kappa)$-bit)

\begin{tabular}{|l|ccccccccc|}
\hline $\begin{array}{l}\text { beakage-rate } \\
\text { Schemes }\end{array}$ & $1 / 8$ & $1 / 6$ & $1 / 4$ & $1 / 3$ & $3 / 8$ & $2 / 5$ & $1 / 2$ & 1 \\
\hline DHLW10 [11] & 94 & 95.2 & 98 & 101.5 & 103.6 & 105 & 112 & - \\
GHV12 [16] & 32 & 32 & 36 & 36 & 40 & 40 & 44 & - \\
NS12 [28] & 36 & - & - & - & - & - & - & - \\
LZSS12 [25] & 18 & 27 & - & - & - & - & - & - \\
Ours & $\mathbf{1 2}$ & $\mathbf{1 2}$ & $\mathbf{1 4}$ & $\mathbf{2 0}$ & $\mathbf{2 4}$ & $\mathbf{3 0}$ & - & - \\
\hline
\end{tabular}

Finally, we give a quantitative comparison among these LR-CCA secure PKE schemes in Table 3 . While for some achievable leakage rate (e.g., $\delta \leq 0.4$ ), our scheme is more efficient compared with the other four schemes. As our construction is general, we can also instantiate it under other standard assumptions, e.g., the DCR assumption [29|10. In [16], the scheme is obtained by applying the CHK transformation [6] to a master-key leakage resilient identity-based encryption scheme. To the best of our knowledge, the constructions of identity-based PKE schemes [16]24 with master-key leakage-resilience are all based on the assumptions (e.g., DLIN) over bilinear groups. Our schemes are the first DDH/DCR based efficient LR-CCA secure PKE schemes with leakage rate 1/2-o(1). 


\section{Instantiation from the DCR Assumption}

Let $N=P Q=\left(2 P^{\prime}+1\right)\left(2 Q^{\prime}+1\right), \widetilde{N}=\widetilde{P} \widetilde{Q}=\left(2 \widetilde{P}^{\prime}+1\right)\left(2 \widetilde{Q}^{\prime}+1\right)$, and the message space be $\{0,1\}^{m}$. Let $\mathfrak{n}, n \in \mathbb{N}$ such that $\mathfrak{n}(\log N-1) \geq \log \widetilde{N}+\lambda+m+\omega(\log \kappa)$ and $n=\lceil\mathfrak{n} \log N / \log \widetilde{N}\rceil$. Let $\left(e k_{\mathrm{CH}}, t d_{\mathrm{CH}}\right) \leftarrow \mathrm{CH} . G e n\left(1^{\kappa}\right)$ sample a chameleon hash function with image set $\{0,1\}^{|\widetilde{N}| / 4}$. Let Ext : $\mathbb{Z}_{N}^{\mathfrak{n}} \times\{0,1\}^{d} \rightarrow\{0,1\}^{m}$ be an average-case $\left(\mathfrak{n}(\log N-1)-\log \widetilde{N}-\lambda, \epsilon_{2}\right)$-strong extractor. Define a map $\chi(y)=b \in \mathbb{Z}_{N}$ for $y \in \mathbb{Z}_{N^{2}}^{*}$, where $y=a+b N \bmod N^{2}(0 \leq a, b \leq N-1)$. The LR-CCA secure PKE from the DCR assumption is presented in Fig. 2, and proof is in the full version.

Key Generation. PKE $2 \cdot \operatorname{Gen}\left(1^{\kappa}\right)$ : Compute $g=-h^{2 N} \bmod N^{2}$ with $h \leftarrow \mathbb{Z}_{N^{2}}^{*}$. Choose $x_{1}, \ldots, x_{\mathfrak{n}} \leftarrow\left\{0, \ldots,\left\lfloor N^{2} / 2\right\rfloor\right\}$ and compute $p k_{i}=g^{x_{i}} \bmod N^{2}$. Choose a random pair $\left(t_{a}^{*}, t_{c}^{*}\right) \leftarrow\{0,1\}^{*} \times \mathcal{R}_{\mathrm{CH}}$ and compute $b^{*}=\mathrm{CH}$.Eval $\left(e k_{\mathrm{CH}}, t_{a}^{*} ; t_{c}^{*}\right)$. Compute $E=\widetilde{g}^{\widetilde{N}^{n}}(1+\widetilde{N})^{-b^{*}} \bmod \widetilde{N}^{n+1}$ with a random $\widetilde{g} \leftarrow \mathbb{Z}_{\widetilde{N}^{n+1}}^{*}$. Return $P K=\left(N, \mathfrak{n}, p k_{1}, \ldots, p k_{\mathfrak{n}}, g, \tilde{N}, n, E, e k_{\mathrm{CH}}\right)$ and $S K=\left(x_{1}, \ldots, x_{\mathfrak{n}}\right)$.

Encryption. $\mathrm{PKE}_{2}$. $\operatorname{Enc}(P K, M)$ : For a public key $P K$ and a message $M \in\{0,1\}^{m}$, choose a random $r \in\{0, \ldots,\lfloor N / 2\rfloor\}$ and a random seed $s \in\{0,1\}^{d}$. It then computes $C=g^{r} \bmod N^{2}, K=\left(\chi\left(p k_{1}^{r}\right), \ldots, \chi\left(p k_{\mathfrak{n}}^{r}\right)\right), \Psi=\operatorname{Ext}(K, s) \oplus M, \Pi=$ $\left(E(1+\widetilde{N})^{b}\right)^{K} \bmod \widetilde{N}^{n+1}$, where $b=\mathrm{CH} . \operatorname{Eval}\left(e k_{\mathrm{CH}}, t_{a} ; t_{c}\right)$ for the auxiliary tag $t_{a}=(C, s, \Psi)$ and a random filter core tag $t_{c} \in \mathcal{R}_{\mathrm{CH}}$. Return $C T=\left(C, s, \Psi, \Pi, t_{c}\right)$.

Note that in the computation of $\Pi, K$ is considered as an element in $\mathbb{Z}_{\widetilde{N}^{n}}$.

Decryption. $\mathrm{PKE}_{2} \cdot \operatorname{Dec}(S K, C T)$, given a ciphertext $C T=\left(C, s, \Psi, \Pi, t_{c}\right)$, computes $K^{\prime}=\left(\chi\left(p k_{1}^{x_{1}}\right), \ldots, \chi\left(p k_{\mathfrak{n}}^{x_{\mathfrak{n}}}\right)\right)$ and $\Pi^{\prime}=\left(E(1+\widetilde{N})^{b}\right)^{K^{\prime}} \bmod \widetilde{N}^{n+1}$, where $b=$ $\mathrm{CH}$.Eval $\left(e k_{\mathrm{CH}},(C, s, \Psi) ; t_{c}\right)$. It checks whether $\Pi=\Pi^{\prime}$. If not, it rejects with $\perp$. Else, it returns $M=\Psi \oplus \operatorname{Ext}\left(K^{\prime}, s\right)$.

Fig. 2. A DCR-based PKE Scheme $\mathrm{PKE}_{2}=\left(\mathrm{PKE}_{2}\right.$.Gen, $\mathrm{PKE}_{2}$.Enc, $\mathrm{PKE} \mathrm{E}_{2}$.Dec $)$

\section{Conclusion and Further Work}

We present a new generic construction of a public-key encryption scheme secure against leakage-resilient chosen-ciphertext attacks, from any $\epsilon$-universal HPS and any one-time lossy filter (OT-LF). Instantiations from the DDH and DCR assumptions show that our construction is practical and achieves leakage rate of $1 / 2-o(1)$. When a slightly weaker universality property of HPS holds with overwhelming probability over the choice of $C$ from the invalid set, LR-CPA security with leakage rate of $1-o(1)$ can be easily constructed from HPS [27]. In our construction, the HPS is required to be $\epsilon$-universal for the worst-case choice of $C$ from the invalid set $\mathcal{C} \backslash \mathcal{V}$. That is the reason why those LR-CPA security with leakage rate of $1-o(1)$ from some HPS cannot be converted into LR-CCA security with OT-LF. The open question is how to further improve leakage rate while keeping the practicality of PKE. 
Acknowledgements. The authors thank anonymous referees for very useful comments and suggestions.

\section{References}

1. Akavia, A., Goldwasser, S., Vaikuntanathan, V.: Simultaneous hardcore bits and cryptography against memory attacks. In: Reingold, O. (ed.) TCC 2009. LNCS, vol. 5444, pp. 474-495. Springer, Heidelberg (2009)

2. Alwen, J., Dodis, Y., Naor, M., Segev, G., Walfish, S., Wichs, D.: Public-key encryption in the bounded-retrieval model. In: Gilbert, H. (ed.) EUROCRYPT 2010. LNCS, vol. 6110, pp. 113-134. Springer, Heidelberg (2010)

3. Brakerski, Z., Goldwasser, S.: Circular and leakage resilient public-key encryption under subgroup indistinguishability - (or: Quadratic residuosity strikes back). In: Rabin, T. (ed.) CRYPTO 2010. LNCS, vol. 6223, pp. 1-20. Springer, Heidelberg (2010)

4. Brakerski, Z., Kalai, Y.T., Katz, J., Vaikuntanathan, V.: Overcoming the hole in the bucket: Public-key cryptography resilient to continual memory leakage. In: FOCS 2010, pp. 501-510. IEEE Computer Society (2010)

5. Camenisch, J., Chandran, N., Shoup, V.: A public key encryption scheme secure against key dependent chosen plaintext and adaptive chosen ciphertext attacks. In: Joux, A. (ed.) EUROCRYPT 2009. LNCS, vol. 5479, pp. 351-368. Springer, Heidelberg (2009)

6. Canetti, R., Halevi, S., Katz, J.: Chosen-ciphertext security from identity-based encryption. In: Cachin, C., Camenisch, J. (eds.) EUROCRYPT 2004. LNCS, vol. 3027, pp. 207-222. Springer, Heidelberg (2004)

7. Chow, S.S.M., Dodis, Y., Rouselakis, Y., Waters, B.: Practical leakage-resilient identity-based encryption from simple assumptions. In: Al-Shaer, E., Keromytis, A.D., Shmatikov, V. (eds.) ACM CCS 2010, pp. 152-161. ACM (2010)

8. Cramer, R., Shoup, V.: A practical public key cryptosystem provably secure against adaptive chosen ciphertext attack. In: Krawczyk, H. (ed.) CRYPTO 1998. LNCS, vol. 1462, pp. 13-25. Springer, Heidelberg (1998)

9. Cramer, R., Shoup, V.: Universal hash proofs and a paradigm for adaptive chosen ciphertext secure public-key encryption. In: Knudsen, L.R. (ed.) EUROCRYPT 2002. LNCS, vol. 2332, pp. 45-64. Springer, Heidelberg (2002)

10. Damgård, I., Jurik, M.: A generalisation, a simplification and some applications of Paillier's probabilistic public-key system. In: Kim, K.-C. (ed.) PKC 2001. LNCS, vol. 1992, pp. 119-136. Springer, Heidelberg (2001)

11. Dodis, Y., Haralambiev, K., López-Alt, A., Wichs, D.: Efficient public-key cryptography in the presence of key leakage. In: Abe, M. (ed.) ASIACRYPT 2010. LNCS, vol. 6477, pp. 613-631. Springer, Heidelberg (2010)

12. Dodis, Y., Kalai, Y.T., Lovett, S.: On cryptography with auxiliary input. In: Mitzenmacher, M. (ed.) STOC 2009, pp. 621-630. ACM (2009)

13. Dodis, Y., Ostrovsky, R., Reyzin, L., Smith, A.: Fuzzy extractors: How to generate strong keys from biometrics and other noisy data. SIAM J. Comput. 38(1), 97-139 (2008)

14. Dziembowski, S., Pietrzak, K.: Leakage-resilient cryptography. In: FOCS 2008, pp. 293-302. IEEE Computer Society (2008)

15. Faust, S., Kiltz, E., Pietrzak, K., Rothblum, G.N.: Leakage-resilient signatures. In: Micciancio, D. (ed.) TCC 2010. LNCS, vol. 5978, pp. 343-360. Springer, Heidelberg (2010) 
16. Galindo, D., Herranz, J., Villar, J.: Identity-based encryption with master keydependent message security and leakage-resilience. In: Foresti, S., Yung, M., Martinelli, F. (eds.) ESORICS 2012. LNCS, vol. 7459, pp. 627-642. Springer, Heidelberg (2012)

17. Halderman, J.A., Schoen, S.D., Heninger, N., Clarkson, W., Paul, W., Calandrino, J.A., Feldman, A.J., Appelbaum, J., Felten, E.W.: Lest we remember: Cold boot attacks on encryption keys. In: van Oorschot, P.C. (ed.) USENIX Security Symposium, pp. 45-60. USENIX Association (2008)

18. Hofheinz, D.: All-but-many lossy trapdoor functions. In: Pointcheval, D., Johansson, T. (eds.) EUROCRYPT 2012. LNCS, vol. 7237, pp. 209-227. Springer, Heidelberg (2012)

19. Hofheinz, D.: Circular chosen-ciphertext security with compact ciphertexts. In: Johansson, T., Nguyen, P.Q. (eds.) EUROCRYPT 2013. LNCS, vol. 7881, pp. 520-536. Springer, Heidelberg (2013)

20. Katz, J., Vaikuntanathan, V.: Signature schemes with bounded leakage resilience. In: Matsui, M. (ed.) ASIACRYPT 2009. LNCS, vol. 5912, pp. 703-720. Springer, Heidelberg (2009)

21. Kiltz, E., Pietrzak, K., Stam, M., Yung, M.: A new randomness extraction paradigm for hybrid encryption. In: Joux, A. (ed.) EUROCRYPT 2009. LNCS, vol. 5479, pp. 590-609. Springer, Heidelberg (2009)

22. Krawczyk, H., Rabin, T.: Chameleon signatures. In: NDSS 2000. The Internet Society (2000)

23. Lai, J., Deng, R.H., Liu, S.: Chameleon all-but-one TDFs and their application to chosen-ciphertext security. In: Catalano, D., Fazio, N., Gennaro, R., Nicolosi, A. (eds.) PKC 2011. LNCS, vol. 6571, pp. 228-245. Springer, Heidelberg (2011)

24. Lewko, A., Rouselakis, Y., Waters, B.: Achieving leakage resilience through dual system encryption. In: Ishai, Y. (ed.) TCC 2011. LNCS, vol. 6597, pp. 70-88. Springer, Heidelberg (2011)

25. Li, S., Zhang, F., Sun, Y., Shen, L.: A new variant of the Cramer-Shoup leakageresilient public key encryption. In: Xhafa, F., Barolli, L., Pop, F., Chen, X., Cristea, V. (eds.) INCoS 2012, pp. 342-346. IEEE (2012)

26. Liu, S., Weng, J., Zhao, Y.: Efficient public key cryptosystem resilient to key leakage chosen ciphertext attacks. In: Dawson, E. (ed.) CT-RSA 2013. LNCS, vol. 7779, pp. 84-100. Springer, Heidelberg (2013)

27. Naor, M., Segev, G.: Public-key cryptosystems resilient to key leakage. In: Halevi, S. (ed.) CRYPTO 2009. LNCS, vol. 5677, pp. 18-35. Springer, Heidelberg (2009)

28. Naor, M., Segev, G.: Public-key cryptosystems resilient to key leakage. SIAM J. Comput. 41(4), 772-814 (2012)

29. Paillier, P.: Public-key cryptosystems based on composite degree residuosity classes. In: Stern, J. (ed.) EUROCRYPT 1999. LNCS, vol. 1592, pp. 223-238. Springer, Heidelberg (1999)

30. Pietrzak, K.: A leakage-resilient mode of operation. In: Joux, A. (ed.) EUROCRYPT 2009. LNCS, vol. 5479, pp. 462-482. Springer, Heidelberg (2009)

31. Peikert, C., Waters, B.: Lossy trapdoor functions and their applications. In: Dwork, C. (ed.) STOC 2008, pp. 187-196. ACM (2008)

32. Rackoff, C., Simon, D.R.: Non-interactive zero-knowledge proof of knowledge and chosen ciphertext attack. In: Feigenbaum, J. (ed.) CRYPTO 1991. LNCS, vol. 576, pp. 433-444. Springer, Heidelberg (1992)

33. Shoup, V.: Sequences of games: a tool for taming complexity in security proofs. Cryptology ePrint Archive, Report 2004/332 (2004), http://eprint.iacr.org/ 